Original Research Paper

\title{
Assessment of Quality of Life, Anxiety and Depression in Children with Turner Syndrome: A Case-Control Study
}

\author{
${ }^{1}$ Khaled Saad, ${ }^{2}$ Abdulrahman Abdullah Al-Atram, \\ ${ }^{3}$ Khaled A. Abdel Baseer, ${ }^{1}$ Ahmed M. Ali and ${ }^{4}$ Amira A. El-Houfey \\ ${ }^{1}$ Department of Pediatrics, Faculty of Medicine, Assiut University, Assiut, Egypt \\ ${ }^{2}$ Department of Psychiatry, College of Medicine, Almajmaah University, KSA \\ ${ }^{3}$ Department of Pediatrics, Faculty of Medicine, South Valley University, Egypt \\ ${ }^{4}$ Department of Community Health Nursing, Assiut University, Assiut, Egypt
}

Article history

Received: 15-01-2015

Revised: 30-01-2015

Accepted: 14-02-2015

Corresponding Author:

Khaled Saad,

Department of Pediatrics,

Faculty of medicine, University of Assiut, Assiut 71516, Egypt Tel: +20-100-608-0182;

Fax: $+20-88-236-8371$

Email: ksaad8@yahoo.com,

Khaled.ali@med.au.edu.eg

\begin{abstract}
Turner syndrome is a genetic disorder that affects only females and is caused by the loss of the whole or a part of the X-chromosome. The aim of this study was to assess the quality of life and frequency of anxiety and depression in a cohort of Egyptian children with Turner Syndrome (TS) diagnosed in Assiut university hospitals, Assiut, Egypt. In a case-control study; 40 females with Turner Syndrome (TS), aged 6-16 years, diagnosed in Assiut university hospitals, Upper Egypt, were compared to 40 agematched apparent healthy girls as controls. They were subjected to full history taking, thorough clinical examination and assessment of pediatric quality of life; we used pediatric anxiety and depression scores. For cognitive assessment, Health-Related Quality of Life (HR-QOL) and psychological evaluation of anxiety and depression; we used the "Children Depression Scale" (CDI), "Children Anxiety Scale" Wechsler Intelligence Scale for Children (WISC) and SF-36 by trained psychiatrists blind to the study. Our study revealed Children Anxiety Scale scores ranged from 13-41 for patients with TS and 5-23 for controls. The mean anxiety scores were significantly higher in TS patients as compared to controls $(p<0.001)$, (27.32 \pm 7.1 versus $15.2 \pm 2.9)$. In addition, we found CDI scores ranged from 38-70 for patients and 31-56 for controls. Mean depression scores were significantly higher in TS patients as compared to controls $(\mathrm{p}<0.001)$, (54.7 \pm 8.8 versus $36.8 \pm 9.9)$. The physical and mental component summary scores of HR-QOL were lower in TS patients when compared to controls. We concluded that female children with TS experience significant problems with HR-QOL, anxiety and depression, which need thorough psychiatric evaluation and rapid intervention of these patients.
\end{abstract}

Keywords: Turner Syndrome, Anxiety, Depression

\section{Introduction}

Turner syndrome is a genetic disorder that affects only females and is caused by the loss of the whole or a part of the X-chromosome (Davies, 2013). It is one of the most common chromosomal aberrations. The similar clinical picture is found with isochromosome (isoXq), ring chromosome $\mathrm{X}$ and deletion of the short or long arm of X chromosome (Donaldson et al., 2006). Monosomy $(45, \mathrm{X})$ accounts for more than $50 \%$ of the karyotypes, while the remaining karyotypes show a mosaic pattern (Davies, 2013; Donaldson et al., 2006). Turner syndrome occurs in approximately 1 in 2,000 live female births
(Donaldson et al., 2006; Saad et al., 2014). The clinical characteristics of Turner syndrome include short stature, infantilism, cubitus valgus, webbed neck, lymphedema, ovarian dysgenesis, renal and cardiovascular anomalies (Donaldson et al., 2006; Saad et al., 2014; Uematsu et al., 2002; Hamelin et al., 2006).

As regards the psychological problems of TS; some researchers had focused on the impact of the physical stigma of TS on psychological development in young women, highlighting short stature, failure of sexual maturity at the same age as their peers, infertility and how these problems relate to psychiatric disorders (Saad et al., 2014; Catinari et al., 2006; Nielsen and Thomsen, 1968). The TS 
personality characterized by excessive anxiety, depressiveness, passivity, distractibility and docility, although no rigorous scientific study has examined these problems (Catinari et al., 2006; Nielsen and Thomsen, 1968). Some studies have used parental evaluations and self-reporting for assessment of psychiatric issues in TS patients. They suggested psychiatric comorbidities especially anxiety and depression in TS patients (Catinari et al., 2006; McCauley et al., 2001; Kilic et al., 2005; Lasaite et al., 2010; Cardoso et al., 2004). In one report; the TS parents reported that TS girls were less socially competent than the controls (McCauley et al., 2001). Two studies (Kilic et al., 2005; Lasaite et al., 2010) reported that TS girls had significantly higher anxiety levels and impaired cognitive functions than age-matched controls. Another large study (Cardoso et al., 2004) reported that 52\% of the TS women met criteria for a current or a past depressive or anxiety disorder and $18 \%$ of TS cases met criteria for psychiatric disorder, including major and minor depression and Anxiety. The majority of the previous studies on TS personality had indistinct mixed statistical data; with sometimes conflict results (Catinari et al., 2006). For these reasons we try in this study to define the frequency of anxiety and depression in children with Turner Syndrome (TS) in a well case-control study.

\section{Patients and Methods}

The Ethical Committee of Assiut University, Assiut, Egypt, approved the study. All methods and procedures used in this study were approved by the Institutional Review Board at Assiut University, Egypt. Informed consent followed the guidelines set forth by the Institutional Review Board at Assiut University and included a brief description of study procedures, a discussion of the voluntary nature of the study, right to withdraw without consequences and confidentiality of information. Informed consents were written in Arabic language that was age appropriate for all participants in the study. Prior to participation in the study, children were required to give their assent and parents were required to give consent for participation. Finally, participants were given a Participant's Bill of Rights. This was a case control study undertaken in the outpatient clinic for children at Assiut University hospitals, Assiut, Egypt.

We conducted a case-control study, in which $40 \mathrm{TS}$ girls documented by karyotyping underwent behavioral assessment for anxiety and depression; compared with 40 apparently healthy females with similar socioeconomic level and age- matched as controls, most of the control girls were sisters of the patients. All control girls had no genetic disorder or chronic illness.

All studied subjects were subjected to the following: full history taking, thorough clinical examination. For cognitive assessment and psychological evaluation of anxiety and depression; we used the following tests by a trained psychiatrists blind to the study:

- "Children Depression Scale (CDI)" test which consists of 27 statements self-rated survey designed to assess all signs of depression in children. The CDI assesses cognitive, behavior and neuro-vegatative signs of depression in children (Kovacs and Beck, 1977)

- Children Anxiety Scale: Children anxiety scale was filled by all the patients and parents. This scale was developed by Castaneda et al. (1956). It consists of 53 items and each item consists of one statement which has two answers yes or no. Total score ranges from (0-53) According to their scores, they were classified into mild, moderate and severe degrees (Castaneda et al., 1956)

- Wechsler Intelligence Scale for Children (WISC) (Wechsler, 2014) was used to assess the IQ for our TS patients and controls

- $\quad$ SF-36 was used to measure Health-Related Quality of Life (HR-QOL). It consists of 36 questions cover 8 health concepts: Physical functioning, role limitations due to physical problems, bodily pain, general health, vitality, social functioning, role limitations due to emotional problems and mental health (Bowling, 1995)

- All patients and controls completed cognitive and psychiatric assessments in neuropsychiatric clinics Assiut University hospital, Assiut, Egypt

\section{Statistical Analysis}

Statistical Package for Social Sciences (SPSS) program version 16 was used for data analysis. Descriptive statistics as minimum, maximum and mean \pm SD and chi-square were used. $P$ value of $\leq 0.05$ denoted the presence of statistically significant difference.

\section{Results}

Table 1 showed the demographic data and HR-QOL results of our Egyptian cohort of girls with TS and controls. We found no significant difference between TS patients and controls as regards age, sex, weight and socioeconomic status. TS subjects had significantly worse, HR-QOL scores in the physical component summary score $(p<0.001)$ and mental component summary score $(p<0.05)$ compared to controls. We found significant correlations between anxiety and depression scores with HR-QOL in TS patients (Table 1). Table 2 showed the clinical feature of TS patients, which included; short stature $(92.5 \%)$, webbed neck $(52.5 \%)$, low posterior hairline $(45 \%)$, cubitus valgus $(30 \%)$, lymphedema (17.5\%), congenital heart diseases (10\%) and endocrine disorders (7.5\%). 
Table 1. The demographic data and HR-QOL results of TS patients and controls

\begin{tabular}{|c|c|c|c|}
\hline & TS patients & Controls & $\mathrm{p}$ - value \\
\hline Number & 40 & 40 & NS \\
\hline Age: Range (years) & $6-16$ & $6-15$ & NS \\
\hline Mean $\pm(\mathrm{SD})$ & $9.32 \pm 2.81$ & $10.01 \pm 1.93$ & NS \\
\hline Sex: Female & 40 & 40 & NS \\
\hline Weight (Mean \pm SD) $(\mathrm{kg})$ & $26.45 \pm 8.66$ & $25.01 \pm 7.61$ & NS \\
\hline Socioeconomic status* & $1.98 \pm 0.93$ & $2.0 \pm 0.87$ & NS \\
\hline $\begin{array}{l}\text { SF-36 PCS (Mean } \pm \text { SD) } \\
\text { (Physical component summary score) }\end{array}$ & $51 \pm 3.8$ & $59 \pm 5.2$ & $<0.001$ \\
\hline $\begin{array}{l}\text { SF-36 MCS (Mean } \pm \text { SD) } \\
\text { (Mental component summary score) }\end{array}$ & $48 \pm 5.4$ & $54 \pm 10.3$ & $<0.05$ \\
\hline Correlation coefficients between HR-QOL scores and anxiety and depression scores & & & \\
\hline Depression scores & 0.65 & 0.15 & $<0.001$ \\
\hline Anxiety scores & 0.54 & 0.17 & $<0.001$ \\
\hline
\end{tabular}

*Socioeconomic status (reported family income divided by median household income for geographical area)

Table 2. Clinical features of studied TS cases

Clinical features (Number)

(Arranged according to frequency)

Short stature (37)

Webbed neck (21) ( $\%$ of cases)

Low posterior hairline

Cubitus valgus (12)

Lymphedema (7)

Short metacarpals (5)

Congenital heart diseases (4)

(4)

Diabetes mellitus (2)

Hypothyroidism (1)

Scoliosis (1)

Table 3. Cognitive profile, anxiety and depression scores of TS cases and controls

\begin{tabular}{llll}
\hline IQ scores & Patients (40) & Controls (40) & $\mathrm{p}$ value \\
\hline Range & $59-119$ & $76-127$ & - \\
Mean \pm SD & $98.9 \pm 15.7$ & $106 \pm 21.5$ & NS \\
Anxiety grades & Number (\%) & Number $(\%)$ & $\mathrm{p}<0.001$ \\
Low & $21(52.5)$ & $34(85)$ & $\mathrm{p}<0.01$ \\
Moderate & $13(32.5)$ & $5(12.5)$ & $\mathrm{p}<0.01$ \\
Severe & $6(15)$ & $1(2.5)$ & $\mathrm{p}<0.01$ \\
Depression grades & & & $\mathrm{p}<0.05$ \\
Minimal & $29(72.5)$ & $38(95)$ & $\mathrm{NS}$ \\
Mild & $6(15)$ & $1(2.5)$ & - \\
High & $3(7.5)$ & $1(2.5)$ & - \\
Severe & $2(5)$ & 0 & - \\
Total & $40(100)$ & $40(100)$ & $\mathrm{p}<0.001$ \\
Anxiety scores & & $5-23$ & \\
Range & $13-41$ & $15.2 \pm 2.9$ & - \\
Mean \pm SD & $27.32 \pm 7.1$ & $31-56$ & $\mathrm{p}<0.001$ \\
Depression scores & & $36.8 \pm 9.9$ & \\
Range & $38-70$ & & \\
Mean \pm SD & $54.7 \pm 8.8$ & & \\
\hline
\end{tabular}

Table 3 showed the Full Scale IQ (FSIQ) of all subjects. We found IQ ranged from 59-119 for patients and 76-127 for controls. Mean FSIQ \pm SD was $98.9 \pm 15.7$ in TS patients versus $106 \pm 21.5$ for controls, with no statistical difference between both groups.
As regards the anxiety scores; we found anxiety scores ranged from 13-41 for TS patients and 5-23 for controls. The mean anxiety scores were significantly higher in TS patients as compared to controls $(p<0.001),(27.32 \pm 7.1$ versus $15.2 \pm 2.9)$. In our study 
TS patients demonstrated a significantly higher incidence of moderate-severe anxiety; 19 versus 6 girls in the control group $(\mathrm{p}<0.01)$ (Table 3$)$.

As regards depression scores; we found depression scores ranged from 38-70 for patients and 31-56 for controls. The mean depression scores were significantly higher in TS patients as compared to controls $(\mathrm{p}<0.001),(54.7 \pm 8.8$ versus $36.8 \pm 9.9)$. In our study TS patients demonstrated a significantly higher incidence of mild to severe grade depression in comparison to the control group $(\mathrm{p}<0.05)$ (Table 3$)$.

\section{Discussion}

The present study enrolled 40 cases with TS. Their ages ranged between 6-16 years. Those patients were diagnosed by karyotype at Assiut university hospitals, Assiut, Upper Egypt. In the present study, we found highly statistical significant differences between patients with TS as compared to controls in different grades of anxiety and depression scores as well as we found the mean values of scores of anxiety and depression were significantly higher among TS patients compared to controls. In addition, TS subjects had significantly worse, HR-QOL scores in physical and mental component summary scores when compared to controls. We found significant correlations between anxiety and depression scores with HR-QOL in TS patients.

Many studies using parental evaluations and selfreport suggested psychiatric problems in girls and adolescents with TS, in the form of immaturity, diminished social relationships, poor self-concept, depression and social anxiety compared to age-matched controls (Saad et al., 2014; Catinari et al., 2006; McCauley et al., 2001; Kilic et al., 2005; Lasaite et al., 2010; Cardoso et al., 2004). These studies deeply investigated the psychopathological comorbidity in TS patients. McCauley et al. (2001) investigated 122 girls with TS and 108 healthy girls as controls. They reported that patients with TS had significantly more social relationships and school progress problems. In addition the parents of TS girls reported that TS girls were less socially competent than the control group (McCauley et al., 2001). Kilic et al. (Kilic et al., 2005) studied 11 TS girls, 9-17 years of age, all with 45,X karyotype. They used CDI, StateTrait Anxiety Inventory for Children (STAIC) and Piers-Harris Children's Self Concept Scale (PHSCS) to assess the extent of depression, anxiety and selfreported self-esteem. They reported that TS girls had significantly lower self-esteem and higher state anxiety levels when compared to control $(\mathrm{p}<0.05)$ (Kilic et al., 2005). Another study showed a lower quality of life, higher tension-anxiety scores and impaired cognitive functions in TS girls than age- matched controls (Lasaite et al., 2010). Cardoso et al. (2004) studied 100 TS patients, according to DSM IV. They reported that; the majority of TS cases who met criteria for a psychiatric condition had a mood or anxiety disorder. Overall, $52 \%$ of the TS women met criteria for a current or a past depressive or anxiety disorder. About $18 \%$ of TS cases met criteria for psychiatric disorder, including major and minor depression, dysthymia and anxiety. About $46 \%$ of TS patients met criteria for a past psychiatric illness, including depression, anxiety and eating disorder (Cardoso et al., 2004).

Our data showed grades of anxiety and depression scores in TS patients higher than the previous reports (Catinari et al., 2006; McCauley et al., 2011; Kilic et al., 2005; Lasaite et al., 2010; Cardoso et al., 2004); this could be explained by the small sample size in our study and the lack of facilities in our developing country which could detect and diagnose TS patients early with the lack of early interventions of any psychological issues in TS patients.

\section{Conclusion}

According to the finding of our study and the previous reports, we can state that TS girls have a higher risk to develop a psychiatric morbidity. In our study; TS patients demonstrated a significantly higher incidence of moderate-severe anxiety and all grades of depression. In addition, the physical and mental component summary scores of HR-QOL were lower in TS patients when compared to controls. We recommend thorough psychiatric evaluation and rapid multidisciplinary intervention of these patients.

The primary limitation of our study was the small sample size; so more randomized controlled trials using a larger number of TS patients with long-term follow-up are needed in the near future.

\section{Funding Information}

The authors have no support or funding to report.

\section{Conflict of Interest}

The authors declare no potential conflicts of interest with respect to the research, authorship and/or publication of this article.

\section{Author's Contribution}

The authors approved the final manuscript as submitted and agree to be accountable for all aspects of the work.

\section{Ethics}

The authors declare no potential conflicts of interest with respect to the research, authorship and/or publication of this article. 


\section{References}

Bowling, A., 1995. Measuring disease: A review of disease-specific quality of life measurement scales. Buckingham: Open University Press.

Cardoso, G., J.R. Daly, N.A. Haq, L. Hanton and D.R. Rubinow et al., 2004. Current and lifetime psychiatric illness in women with Turner syndrome. Gynecol. Endocrinol., 19: 313-319.

Castaneda, A., B.R. McCandless and D.S. Palermo, 1956. The children's form of the manifest anxiety scale. Child Development, 27: 317-326.

Catinari, S., A. Vass and U. Heresco-Levy, 2006. Psychiatric manifestations in Turner Syndrome: A brief survey. Isr. J. Psychiatry Relat. Sci., 43: 293-295.

Davies, W., 2013. Using mouse models to investigate sex-linked genetic effects on brain, behaviour and vulnerability to neuropsychiatric disorders. Brain Res Bull., 92: 12-20.

DOI: $10.1016 /$ j.brainresbull.2011.06.018

Donaldson, M.D.C., E.J. Gault, K.W. Tan and D.B. Dunger, 2006. Optimising management in Turner syndrome: From infancy to adult transfer. Arch. Dis. Child, 91: 513-520.

DOI: $10.1136 /$ adc.2003.035907

Hamelin, C.E., G. Anglin, C.A. Quigley and C.L. Deal, 2006. Genomic imprinting in Turner syndrome: Effects on response to growth hormone and on risk of sensorineural hearing loss. J. Clin. Endocrinol. Metab., 91: 3002-310. DOI: $10.1210 /$ jc. 2006-0490

Kilic, B.G., A.T. Ergur and G. Ocal, 2005. Depression, levels of anxiety and self-concept in girls with Turner's syndrome. J. Pediatr. Endocrinol. Metab., 18: 1111-1117.

DOI: 10.1515/JPEM.2005.18.11.1111
Kovacs, M. and A. Beck, 1977. An Empirical-Clinical Approach toward a Definition of Childhood Depression. In: Depression in Childhood: Diagnosis, Treatment and Conceptual Models, Schulterbrandt J.G. and A. Raskin (Eds.). Washington, DC, USA: Department of Health, Education and Welfare, pp: 1-26.

Lasaite, L., D. Lasiene and L. Lasas, 2010. Cognition, emotions and quality of life in Lithuanian girls with Turner syndrome after growth hormone therapy discontinuation. J. Pediatr. Endocrinol. Metab., 23: 443-450. DOI: 10.1515/jpem.2010.075

McCauley, E., P. Feuillan, H. Kushner and J.L. Ross, 2001. Psychosocial development in adolescents with Turner syndrome. J. Dev. Behav. Pediatr., 22: 360-365. DOI: 10.1097/00004703-200112000-00003

Nielsen, J. and N. Thomsen, 1968. A psychiatriccytogenetic study of a female patient with $45 / 46 / 47$ chromosomes and sex chromosomes XO/XX/XXX. Acta Psychiatr. Scand., 44: 141-155. DOI: 10.1111/j.1600-0447.1968.tb07501.x

Saad, K., A.A. Abdelrahman, Y.F. Abdel-Raheem, E.R. Othman and R. Badry et al., 2014. Turner syndrome: Review of clinical, neuropsychiatric and EEG status: An experience of tertiary center. Acta Neurol. Belg., 114: 1-9. DOI: $10.1007 / \mathrm{s} 13760-013-0264-9$

Uematsu, A., T. Yorifuji, J. Muroi, M. Kawai and M. Mamada et al., 2002. Parental origin of normal X chromosomes in Turner syndrome patients with various karyotypes: Implications for the mechanism leading to generation of a $45, \mathrm{X}$ karyotype. Am. J. Med. Genet., 111: 134-139. DOI: 10.1002/ajmg.10506

Wechsler, D., 2004. The Wechsler Intelligence Scale for Children. 4th Edn., London: Pearson Assessment. 